\title{
Partly burned white dwarf survivors from peculiar thermonuclear supernovae
}

\section{Roberto Raddi*}

Dr Remeis-Sternwarte, ECAP, Friedrich Alexander Universität Erlangen-Nürnberg, Sternwartstr. 7, 96049 Bamberg, Germany

E-mail: roberto.raddiefau.de

LP 40-365 is the prototype of a new class of runaway stars, thus far consisting of four members. The atmospheres of the known "LP 40-365 stars" are dominated by oxygen, neon, and magnesium, and are sprinkled with the ashes of partial oxygen and silicon burning. Possessing masses and radii in the range of $0.2-0.3 \mathrm{M}_{\odot}$ and $0.16-0.6 \mathrm{R}_{\odot}$, respectively, the LP 40-365 stars are suggested to be the former accreting white dwarfs that have survived disruption in nearChandrasekhar-mass thermonuclear supernova explosions. Due to remarkable mass loss, the LP 40-365 stars were violently unbound from their companions. Detailed spectroscopic and kinematic analysis is crucial to assess their physical properties, which can be used as boundary conditions for population synthesis modelling aiming to characterise the formation and evolution mechanisms.

The Golden Age of Cataclysmic Variables and Related Objects V (GOLDEN2019)

2-7 September 2019

Palermo, Italy

${ }^{*}$ Speaker. 


\section{Introduction}

Kinematically peculiar stars are messengers of some of the most violent astrophysical phenomena, such as supernova explosions. The complete disruption of a white dwarf, as it is caused by a thermonuclear explosion (i.e. SN Ia), naturally frees the companion star imparting a large ejection velocity that is equal to the orbital motion at the moment of explosion [10, 21]. Recently, three new high-velocity stars, with unusual carbon-oxygen atmospheres, were dug out of the Gaia database $[3,4]$. These three stars have been suggested as the former white dwarf donors that were ejected from compact binaries, where the primary stars exploded in a specific SN Ia scenario, described as dynamically driven double-degenerate double-detonation (D6; [19, 20]).

Stepping back to before the second data release of Gaia, the high-proper motion white dwarf candidate LP 40-365 [13, 5] was discovered to have a unique atmosphere, mostly made of neon, oxygen, and magnesium, devoid of hydrogen and helium, and also containing abundant traces of partial silicon-burning products $[17,14]$. Owing to this unusual composition and a radial velocity of $\approx 500 \mathrm{~km} \mathrm{~s}^{-1}$, LP 40-365 was suggested to be the former accreting white dwarf that survived its disruption in a peculiar supernova explosion [17], of the likes of SN 2002-cx (SN Iax; [12, 7, 2]).

The unusual atmosphere of LP 40-365 indicates a low surface-gravity $(\log g \approx 5.5)$ and $T_{\text {eff }} \approx$ $10,000 \mathrm{~K}[17,16]$. This star is $630 \mathrm{pc}$ away from the Sun and is fleeing the Milky Way with a rest-frame velocity of $850 \mathrm{~km} \mathrm{~s}^{-1}$, as constrained by a precise Gaia parallax [15]. With a radius of $0.16 \mathrm{R}_{\odot}, \mathrm{LP} 40-365$ is brighter than canonical white dwarfs, but it is also fainter than mainsequence stars of similar temperature [15], suggesting it could be "puffed up", due to an increase of internal energy that occurred during the failed supernova explosion $[9,11]$. The surface gravity and radius of LP 40-365 constrain its mass to $\sim 0.3 \mathrm{M}_{\odot}$, implying that about $1 \mathrm{M}_{\odot}$ was dispersed as consequence of the supernova explosion. Such extreme mass loss led to the separation from its former donor star, disrupting the original binary [6]. The ejection velocity achieved by LP 40-365 is in the range of $600 \mathrm{~km} \mathrm{~s}^{-1}$ [15].

\section{The class of $L P 40-365$ stars}

Follow-up spectroscopy of Gaia sources has revealed two other, kinematically peculiar stars that possess an atmospheric composition mirroring that of LP 40-365 [16]. Their atmospheres are dominated by the alpha elements, neon (60-65\%), oxygen (30\%), and magnesium (3-10\%). Other trace elements account for $\simeq 0.1 \%$ of the atmospheric mass. Notably titanium, chromium, manganese, and nickel are present with super-Solar composition with respect to iron, as expected for near-Chandrasekhar mass explosions. The abundance pattern of the oxygen and silicon burning products, which are detected within the LP 40-365 stars, broadly agrees with the expected composition of partly burned remnants resulting from the pure deflagrations of carbon-oxygen and oxygen-neon core white dwarfs $[1,8]$.

The first star, J1603-6613, is a spectroscopic clone of LP40-365, having the same temperature and surface gravity; this star is also unbound from the Milky Way. The second star, $\mathrm{J} 1825-3757$, is $\approx 3000 \mathrm{~K}$ hotter than the other two and it is intrinsically bright, due to a larger radius of $0.6 \mathrm{M}_{\odot}$; this star is bound to the Milky Way, as it travels onto a retrograde orbit. The three 
stars have rather similar masses $\left(0.2-0.3 \mathrm{M}_{\odot}\right)$, and their space velocities suggest that the same formation and ejection mechanisms took place.

In addition to these three stars with precise Gaia astrometry, on more has been found within the Sloan spectroscopic database, J0905-2510. Although a parallax is not yet available, its spectrum shows distinctive features identifying it as another member of the LP 40-365 star family [16].

\section{An outlook to the population}

The LP40-365 stars have well-defined chemical, physical, and kinematic properties that make them unlike any other stars. Nevertheless, there are several open questions that remain unanswered and are of fundamental importance in order to constrain their formation and evolution mechanisms.

Despite their chemical homogeneity, the bulk atmospheric composition of LP 40-365 stars is at odds with respect to theoretical predictions [16], as they display either too much carbon or too much oxygen and neon, which do not match the expected nucleosynthetic yields in case of carbon-oxygen or oxygen-neon core white dwarf progenitors, respectively [1, 8].

Due to the sudden increase of internal energy, white dwarfs that survive to thermonuclear explosions are expected to suddenly expand and rapidly radiate away their energy as they cool down $[9,11,18]$. Nevertheless, the full cooling process may take up to a few hundred million years, and it could be influenced by the atmospheric composition as well as the internal structure of these stars that is yet unknown. The expanded radii of the LP 40-365 stars suggest that they have not yet reached equilibrium and are still evolving, implying their ages could, at least, be as long as a few tens of million years [22]. The size of the Galaxy offers an upper limit for the age of LP 40-365, as this star would fly across the entire Milky Way in $\sim 150 \mathrm{Myr}$ due to its high rest-frame velocity. This upper limit is compatible with theoretical predictions [22], but the age of all class members could be much younger than that, if they were expelled from closer distances within the Galactic disc [16].

In order to further characterise the population of LP 40-365 stars, it has been suggested they are born with the rate of SN Iax [16] (i.e. 10\% of normal SN Ia [2]). Such a toy-model suggests that around 20 LP 40-365 stars could be detectable by the end of the Gaia mission, with $G<19$, within $2 \mathrm{kpc}$ from the Sun [16]. If the stars so-far identified are the fastest of their kind, the dominant population should be made of much slower objects that could be harder to find.

The global picture may be more complex. In fact, it should be noted that there could also be LP 40-365 stars that did not get separated from their companions or others that already descended onto the cooling tracks of low-mass white dwarfs.

Future observational projects are fundamental to identify new members of this class. Detailed theoretical work, involving population synthesis and hydrodynamical simulations of peculiar thermonuclear explosions, is also key for interpreting the properties of these stars, in order to constrain their formation and evolution.

\section{DISCUSSION}

J. Isern: There are other examples of "iron"-core white dwarfs, as told by the mass-radius relationship, that could have a similar origin. Can you comment on that? 
R. Raddi's comment: Several canonical white dwarfs, i.e. characterised by more typical atmospheres, have been proposed to host Fe-cores. Unfortunately the magnitude of error bars is compatible with the mass-radius relation for a range of core compositions.

A. Tanikawa: What is the mass of LP $40-365$ stars?

R. Raddi's comment: Their masses range between $0.2-0.3 M_{\odot}$. We inferred them by combining their $\log g$ and radii, which are measured from their spectra and the angular diameter, respectively.

\section{References}

[1] Fink, M., et al. 2014. Three-dimensional pure deflagration models with nucleosynthesis and synthetic observables for Type Ia supernovae. Monthly Notices of the Royal Astronomical Society 438, 1762.

[2] Foley, R. J., et al. 2013. Type Iax Supernovae: A New Class of Stellar Explosion. The Astrophysical Journal 767, 57.

[3] Gaia Collaboration, et al. 2016. The Gaia mission. Astronomy and Astrophysics 595, A1.

[4] Gaia Collaboration, et al. 2018. Gaia Data Release 2. Summary of the contents and survey properties. Astronomy and Astrophysics 616, A1.

[5] Giclas, H. L., Burnham, R., Thomas, N. G. 1970. A list of white dwarf suspects III: special objects of small proper motion from the Lowell survey. Lowell Observatory Bulletin 7, 183.

[6] Hills, J. G. 1983. The effects of sudden mass loss and a random kick velocity produced in a supernova explosion on the dynamics of a binary star of arbitrary orbital eccentricity. Applications to X-ray binaries and to the binary pulsars. The Astrophysical Journal 267, 322.

[7] Jha, S., et al. 2006. Late-Time Spectroscopy of SN 2002cx: The Prototype of a New Subclass of Type Ia Supernovae. The Astronomical Journal 132, 189.

[8] Jones, S., et al. 2019. Remnants and ejecta of thermonuclear electron-capture supernovae. Constraining oxygen-neon deflagrations in high-density white dwarfs. Astronomy and Astrophysics 622, A74.

[9] Jordan, G. C., Perets, H. B., Fisher, R. T., van Rossum, D. R. 2012. Failed-detonation Supernovae: Subluminous Low-velocity Ia Supernovae and their Kicked Remnant White Dwarfs with Iron-rich Cores. The Astrophysical Journal 761, L23.

[10] Justham, S., Wolf, C., Podsiadlowski, P., Han, Z. 2009. Type Ia supernovae and the formation of single low-mass white dwarfs. Astronomy and Astrophysics 493, 1081.

[11] Kromer, M., et al. 2013. 3D deflagration simulations leaving bound remnants: a model for 2002cx-like Type Ia supernovae. Monthly Notices of the Royal Astronomical Society 429, 2287.

[12] Li, W., et al. 2003. SN 2002cx: The Most Peculiar Known Type Ia Supernova. Publications of the Astronomical Society of the Pacific 115, 453.

[13] Luyten, W. J. 1970. Proper Motion Survey with the forty-eight inch Schmidt telescope. The zone +70 to +75 . Proper Motion Survey with the forty-eight inch Schmidt telescope.

[14] Raddi, R., et al. 2018. Further Insight on the Hypervelocity White Dwarf, LP 40-365 (GD 492): A Nearby Emissary from a Single-degenerate Type Ia Supernova. The Astrophysical Journal 858, 3. 
[15] Raddi, R., et al. 2018. Anatomy of the hyper-runaway star LP 40-365 with Gaia. Monthly Notices of the Royal Astronomical Society 479, L96.

[16] Raddi, R., et al. 2019. Partly burnt runaway stellar remnants from peculiar thermonuclear supernovae. Monthly Notices of the Royal Astronomical Society 489, 1489.

[17] Vennes, S., et al. 2017. An unusual white dwarf star may be a surviving remnant of a subluminous Type Ia supernova. Science 357, 680.

[18] Shen, K. J., Schwab, J. 2017. Wait for It: Post-supernova Winds Driven by Delayed Radioactive Decays. The Astrophysical Journal 834, 180.

[19] Shen, K. J., Kasen, D., Miles, B. J., Townsley, D. M. 2018. Sub-Chandrasekhar-mass White Dwarf Detonations Revisited. The Astrophysical Journal 854, 52.

[20] Shen, K. J., et al. 2018. Three Hypervelocity White Dwarfs in Gaia DR2: Evidence for Dynamically Driven Double-degenerate Double-detonation Type Ia Supernovae. The Astrophysical Journal 865, 15.

[21] Wang, B., Han, Z. 2009. Companion stars of type Ia supernovae and hypervelocity stars. Astronomy and Astrophysics 508, L27.

[22] Zhang, M., Fuller, J., Schwab, J., Foley, R. J. 2019. The Long-term Evolution and Appearance of Type Iax Postgenitor Stars. The Astrophysical Journal 872, 29. 